\title{
Tratamiento de la sialorrea con toxina botulinica en niños con paralisis cerebral
}

Treatment of sialorrhea with botulinum toxin in children with cerebral palsy

\author{
Emma Rivera La Plata ${ }^{1, a}$, Doris Reymer Reinoso ${ }^{2, b}$, Helga Solis Mujica ${ }^{3,4, c}$
}

\section{RESUMEN}

Objetivo: Determinar la utilidad de la toxina botulínica en el tratamiento de la sialorrea en niños con Parálisis Cerebral. Material y Métodos: Estudio Prospectivo experimental, longitudinal de cohorte y abierto. Seis sujetos entre 6 y 14 años de edad con parálisis cerebral y sialorrea moderada a severa. Los sujetos que presentaban sialorrea moderada fueron inyectados con 5 unidades de toxina botulínica y sialorrea severa 10 unidades de toxina botulínica en glándula submaxilar y parotida. Las inyecciones realizadas bajo localización por ultrasonido. Cuantificación del babeo usando escala de frecuencia y severidad. Escala análoga visual para determinar grado de salivación y escala de nivel de satisfacción post-inyección. Se evaluó la deglución pre y post inyección con una evaluación por terapista de lenguaje. Resultados: Todos los sujetos fueron inyectados exitosamente con toxina botulínica A sin complicaciones sistémicas o locales. No hubo efectos adversos en la deglución. La cuantificación de babeo fue más indicativa con la Escala análoga visual. El efecto beneficioso del tratamiento se observo entre el tercer y séptimo día, solo un caso no mejoro sino después del primer mes. Conclusiones: La Toxina botulínica A es un tratamiento minimamente invasivo y potencialmente seguro y promisorio para la sialorrea en niños con Parálisis Cerebral. PALABRAS CLAVE: Sialorrea, toxina botulínica tipo A, parálisis cerebral.

\section{SUMMARY}

Objective: To determine the utility of botulinum toxin in the treatment of sialorrhea in children with Cerebral Palsy. Material and Methods: Experimental, longitudinal, cohort and open prospective study. Six subjects between 6 and 14 years of age with cerebral palsy and moderate to severe sialorrhea. Subjects presenting moderate sialorrhea were injected with 5 units of botulinum toxin and severe sialorrhea, 10 units of botulinum toxin in the submaxillary and parotid glands. Injections performed under ultrasound localization. Quantification of drooling using frequency and severity scale. Visual analog scale to determine degree of salivation and level of satisfaction level post-injection. Swallowing pre and post injection was evaluated with an evaluation by speech therapist. Results: All subjects were successfully injected with botulinum toxin A without systemic or local complications. There were no adverse effects on swallowing. Drool quantification was more indicative with the Visual Analog Scale. The beneficial effect of the treatment was observed between the third and seventh day, only one case did not improve until after the first month. Conclusions: Botulinum toxin A is a minimally invasive and potentially safe and promising treatment for sialorrhea in children with Cerebral Palsy.

KEYWORDS: Sialorrhea, botulinum toxin, cerebral palsy.

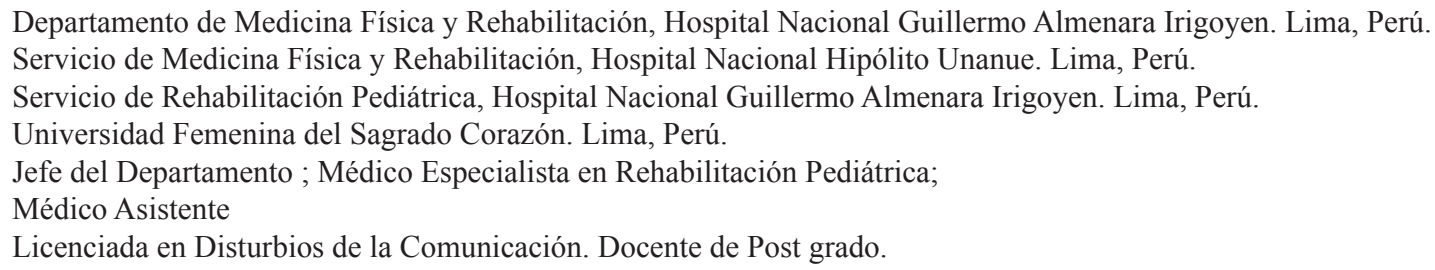


Rev Hered Rehab. 2019; 1:27-35.

\section{INTRODUCCIÓN}

La Sialorrea se considera como una secuela de lesión neurológica, es un factor frecuente que favorece a la discapacidad de las personas 2,4 , la prevalencia del babeo perturbador en niños con parálisis cerebral es de 10 a $37 \%$ (1).

La Sialorrea en pacientes con Parálisis Cerebral puede ser considerada como su peor aflicción aún en niños moderadamente afectados. Tiene implicancias psicosociales, la sialorrea actúa como una barrera física y emocional, también desarrolla una morbilidad orgánica que incluye excoriaciones de la piel, infecciones micóticas, aspiración de saliva y/o deshidratación (2).

Las numerosas terapias tanto médicas como quirúrgicas no han dado resultados universalmente exitosos, se han reportado estudios en el uso de toxina botulínica tipo A con resultados óptimos en el tratamiento de la sialorrea en individuos con daño neurológico (3).

Las inyecciones con toxina botulínica tipo A han provocado seguridad y efectividad. Los estudios sobre toxina botulínica tipo A se centran sus efectos a nivel de la función neuromuscular, con éxito en el tratamiento de la función autonómica, como inhibidor efectivo de las glándulas salivales, es un tratamiento de gran potencial para la sialorrea (4).

La toxina botulínica ha sido inyectada dentro de las glándulas salivales en adultos con enfermedad de parkinson y esclerosis lateral amiotrófica en niños presentan pocos efectos colaterales con una mejoría potencial para la sialorrea por encima de los 4-5 meses (5).

Nuestro objetivo fue evaluar y demostrar la utilidad de toxina botulínica A en el tratamiento de la sialorrea en niños con parálisis cerebral, así como evaluar el nivel de satisfacción de los resultados obtenidos en sialorrea.

Si logramos que estos niños disminuyan su babeo, facilitara el mejor desenvolvimiento social, mejorando la auto confianza del niño, así como facilitar el cuidado de ellos, por lo tanto, mejoraremos calidad de vida en estos niños.

\section{MATERIAL Y METODOS}

El presente trabajo es un estudio prospectivo cuasi experimental, longitudinal de cohorte y abierto, ya que se interviene o se manipula la variable independiente (toxina botulínica) a fin de observar el efecto sobre la sialorrea, donde se evaluaron y trataron 06 pacientes afectados por Parálisis Cerebral y sialorrea moderada a severa, fueron vistos en el periodo de octubre del 2013 a marzo del 2014 en el Servicio de Patología del Desarrollo del Departamento de Medicina Física y Rehabilitación del Hospital Nacional Guillermo Almenara Irigoyen, donde se les invitó a participar en el estudio tras consentimiento informado por parte de los padres.

\section{Criterios de Inclusión:}

1. Pacientes con Parálisis Cerebral que presentan sialorrea de moderada a severa.

2. Sujetos entre 6 a 14 años de edad.

Tabla 1. Valoración de la sialorrea en nuestros pacientes, escala de severidad y frecuencia.

\begin{tabular}{ccc}
\hline & Antes del tratamiento & A los 30 días \\
\hline Paciente 1 & & 3 \\
Severidad & 4 & 3 \\
Frecuencia & 5 & \\
Paciente 2 & & 3 \\
Severidad & 3 & 2 \\
Frecuencia & 4 & 3 \\
Paciente 3 & & 3 \\
Severidad & 4 & \\
Frecuencia & 4 & 2 \\
Paciente 4 & & 1 \\
Severidad & 3 & 2 \\
Frecuencia & 2 & 1 \\
Paciente 5 & & \\
Severidad & 3 & 2 \\
Frecuencia & 3 & 1 \\
Paciente 6 & & \\
Severidad & 3 & \\
Frecuencia & 2 & \\
\hline
\end{tabular}

Tabla 2. Sialorrea: escala análoga visual

\begin{tabular}{ll}
\hline EVA & SALIVACIÓN \\
\hline $0-20$ & Normal \\
$21-40$ & Rebalce de Boca \\
$41-60$ & Babeo mínimo \\
$61-80$ & Babeo moderado \\
$81-100$ & Babeo constante \\
\hline
\end{tabular}


Rev Hered Rehab. 2019; 1:27-35.

Tabla 3. Escala de clasificación de los pacientes de la frecuencia y severidad

\author{
Frecuencia \\ $1=$ Nunca \\ $2=$ Ocasionalmente (no todo el día) \\ $3=$ Frecuentemente (parte del día) \\ $4=$ Constantemente \\ Severidad \\ $1=$ Seco (ningún babeo) \\ $2=$ Mediano (sólo labios mojados) \\ $3=$ Moderado (labios y babero mojados) \\ $4=$ Severo (babeo que se extiende a la ropa) \\ $5=$ Profuso (manos, cubeta y objetos mojados)
}

Tabla 4. Escala de clasificación del nivel de satisfaccion post-inyeccion

Sobre todo, ¿cómo tiene el babeo desde la inyección con Botox?

Marcadamente peor

Moderadamente peor

Levemente peor

No cambios

Leve mejoría

Mejoría moderada

Mejoría marcada

Si ha visto mejoría, ¿cuánto ha mejorado para usted?

¿Cómo podría clasificar su nivel de satisfacción con el tratamiento para el babeo?

Marcadamente insatisfecho

Moderadamente insatisfecho

Levemente insatisfecho

Levemente satisfecho

Moderadamente satisfecho

Marcadamente satisfecho

\section{Criterios de Exclusión:}

1. Presencia de disfagia en evaluación por Terapeuta del Lenguaje.

2. Sujetos que han tenido cirugía de la glándula Submaxilar.

3. Alergia conocida o sensibilidad para la medicación en estudio o sus componentes.

Los pacientes fueron $6(100 \%)$ sexo masculino. El rango de edad fue de 6 a 14 años con una edad media de 11 años. Se realizó una historia clínica completa, poniendo énfasis en medicaciones y alergias.

Se realizo una evaluación por terapista de lenguaje pre y post-inyección, para descartar la evidencia de disfagia o trastornos en la deglución.
Las evaluaciones de los cambios de sialorrea fueron realizadas con escala análoga visual de babeo, que es una escala verbal simple conformada por 5 categorías (tabla 2), donde cero indica salivación normal y 100 indica el máximo de salivación 8 . La escala de clasificación de la frecuencia y severidad de la sialorrea originalmente empleada por Crysdale et al., (4) (tabla 3), y la escala de clasificación de sialorrea post-inyección para determinar el nivel de satisfacción empleada por Dana et al., (5) (tabla 4), se emplearon las pruebas no paramétricas de Wilcoxon y Fredman, ya que los datos presentan variables ordinales, además se contó con pocos casos.

Se aplica 5 unidades de toxina botulínica a los pacientes con sialorrea moderada y 10 unidades a los pacientes con sialorrea severa. Los criterios de selección de dosis proceden de los casos descritos en la literatura, los pacientes seleccionados eran sometidos a la aplicación de toxina botulínica A (Botox ${ }^{\circledR}$, Allergan Pharmaceuticals), la dilución utilizada fue de 100 unidades en $2 \mathrm{ml}$ de suero salino al 0,9\%. la técnica usada para la inyección fue la siguiente:

Se aplicó una crema anestésica tópica EMLA(crema anestésica, astra Zeneca LP) por aproximadamente 60 minutos en la piel encima de las glándulas submaxilar y/o parótida.

Se localizo las glándulas por guía de ultrasonido con el transductor lineal XP Acuson.

Se usó una aguja $26 \mathrm{G} \mathrm{x1/2"} \mathrm{y} \mathrm{una} \mathrm{jeringa} \mathrm{de} 1 \mathrm{ml}$. Para la aplicación del medicamento.

Se inyecto $5 \mathrm{U}$ o $10 \mathrm{U}$ de toxina botulínica $\mathrm{A}$ en cada glándula submaxilar y parótida, según severidad (figura 1 y figura 2).

Se observó a los niños una hora después del procedimiento para observar efectos adversos.

Se realizaron evaluaciones post-inyección al 3er día, 7mo día, 15avo día y al mes de la aplicación, donde se evaluó las variaciones de la sialorrea, además se indago por algún efecto adverso o si hubo alguna ocurrencia en este periodo (anexo 1).

\section{RESULTADOS}

Se incluyeron al estudio seis niños. Todos fueron sometidos a seguimiento hasta el final del estudio que 


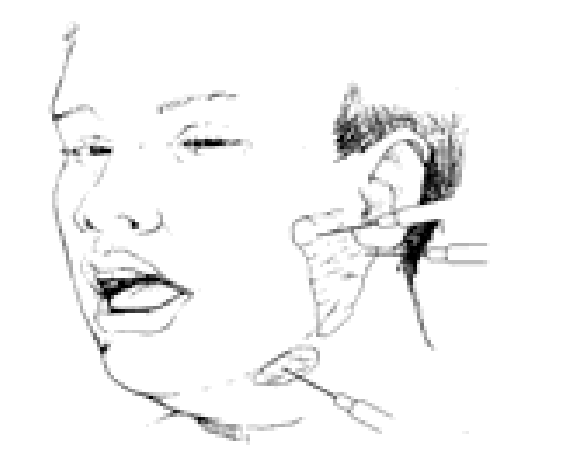

Figura 1. Esquema de puntos de inyección de toxina botulínica en glándula submaxilar y parótida.

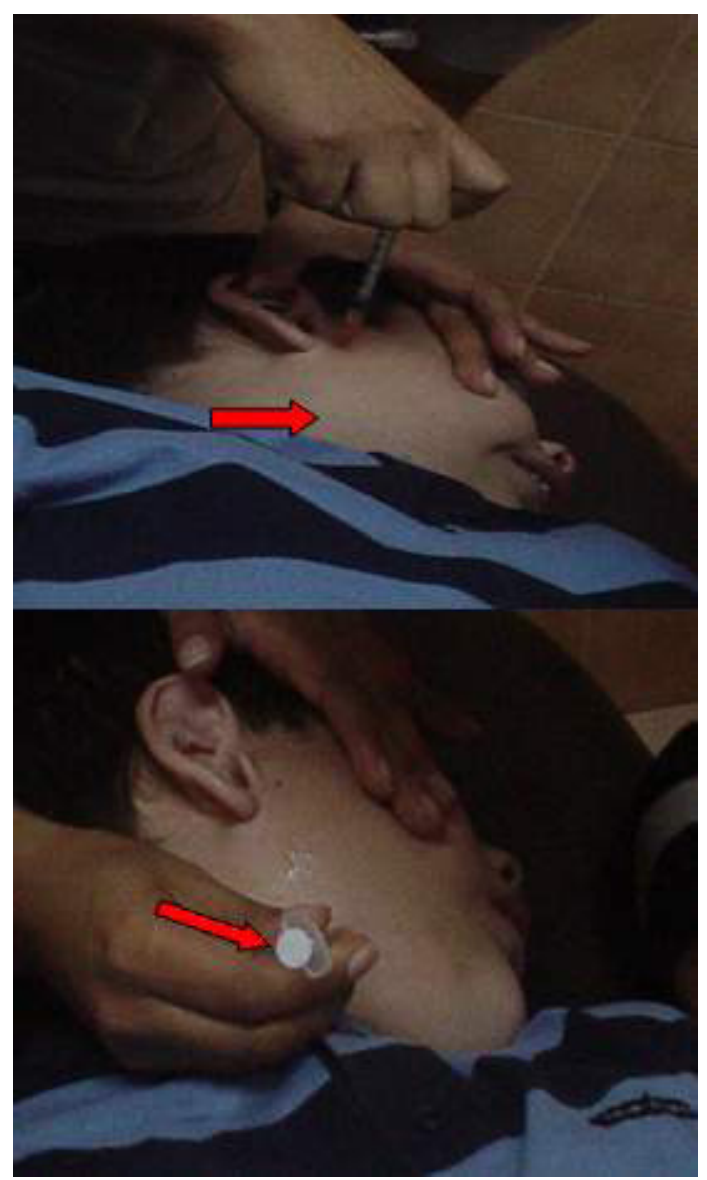

Figura 2. Aplicación de la inyección de toxina botulínica en glándula submaxilar y parótida.

fue de un mes. Hubo seis niños con un rango de edad de 6 a 14 años. Todos tuvieron diagnóstico de parálisis cerebral de diversa etiología, asociado a retardo mental leve con buena colaboración. Tres niños presentaban sialorrea moderada y frecuentemente durante el día, catalogados como babeadores moderados, Tres niños presentaban sialorrea severa a profusa constantes $\mathrm{y}$ fueron catalogados como severos.

Los parientes fueron interrogados para informar sobre si ellos sentían que el tratamiento fue beneficioso y si podrían considerar una nueva aplicación. Tres pacientes $(50 \%)$ sintieron una mejoría marcada en la sialorrea y expresaron su marcada satisfacción por la aplicación de toxina botulínica a sus niños.

Se determino una evolución satisfactoria por medio de una reducción significativa y progresiva de la sialorrea en todas las evaluaciones con la escala análoga visual, no así con la escala de severidad y frecuencia donde se aprecia diferencia significativa al mes de evaluación (tabla 1).

A los treinta días después del tratamiento, disminuyo significativamente la sialorrea, ya que la variable dependiente EVA, así lo indica, también se comprueba esta diferencia significativa con los indicadores de Frecuencia y severidad de sialorrea con un $95 \%$ de confianza con una $\mathrm{p}=0.025<0.05$ (Prueba de Wilcoxon).

\section{Paciente 1}

Paciente de 12 años de edad, diagnosticado con Parálisis Cerebral tipo cuadriparesia espástica, las quejas por sialorrea severa limitaban al paciente y al familiar en sus diferentes actividades de vida diaria. La sialorrea era constantemente frecuencia grado 4 y severidad grado 5, el babeo era profuso mojaba manos, ropa y objetos de su entorno, ocasionalmente presentaba enrojecimiento del pie, catalogado como babeador severo. Escala análoga visual 100. Fueron aplicadas 10 unidades de toxina en glándula submaxilar y parotida de cada lado, obteniéndose una respuesta terapéutica a los 7 días de tratamiento con un 30\% de mejoría. Refiriendo el familiar una leve satisfacción por el procedimiento. A los 30 días postaplicación se observo una mejoría del $35 \%$ con babeo mínimo, mojando escasamente la ropa con una frecuencia y severidad de 3, su EVA de 65.

\section{Paciente 2}

Paciente de 10 años de edad, diagnosticado con Parálisis Cerebral tipo cuadriparesia espástica asociado a síndrome convulsivo, las quejas por sialorrea perturbaban en forma moderada-severa a la persona que lo cuidaba en su alimentación y actividades fuera 
Rev Hered Rehab. 2019; 1:27-35.

de la casa. La sialorrea era frecuentemente frecuencia grado 3 y severidad grado 4 , ocasionalmente presentaba enrojecimiento de la piel, catalogado como babeador severo. Escala análoga visual 80 . Fueron aplicada 10 unidades de toxina en glándula submaxilar y parótida de cada lado, las quejas de hipersalivación por parte del familiar después del procedimiento se habían incrementado, obteniéndose una respuesta terapéutica favorable a partir del mes de tratamiento, con una mejoría del $40 \%$ con una sialorrea en frecuencia grado 3 y severidad grado 2, con una EVA de 56.

\section{Paciente 3}

Paciente de 9 años de edad, diagnosticado con Parálisis Cerebral tipo cuadriparesia espástica, asociado a retardo leve de buena colaboración, las quejas por sialorrea severa limitaban al paciente y al familiar en sus diferentes actividades vida diaria. La sialorrea era constantemente frecuencia grado $4 \mathrm{y}$ severidad grado 4 y el babeo era profuso mojaba manos, ropa y objetos de su entorno, ocasionalmente presentaba enrojecimiento de la piel, catalogado como babeador severo. Escala análoga visual 90. Fueron aplicadas 10 unidades de toxina en glándula submaxilar y parotida de cada lado, obteniéndose una respuesta terapéutica a los 7 días de tratamiento. Refiriendo el familiar una leve satisfacción por el procedimiento. A los 30 días postaplicación se observo una mejoría del $50 \%$ con un babeo mínimo, mojando escasamente la ropa con una frecuencia y severidad de 3, su EVA de 65.

\section{Paciente 4}

Paciente de 14 años de edad, diagnosticado con Parálisis Cerebral tipo cuadriparesia espástica, las quejas por hipersalivación perturbaban moderadamente la auto confianza del paciente, así como su alimentación en forma moderada a severa. La sialorrea era frecuentemente con una frecuencia grado 3 y severidad grado 3 , como resultado del babeo realizaba un cambio al día de polo (durante la alimentación) y ocasionalmente presentaba enrojecimiento de la piel. Escala análoga visual 60. Fueron aplicadas 5 unidades de toxina en glándula submaxilar y parotida de cada lado, obteniéndose una clara respuesta terapéutica a los 3 días de tratamiento con una mejoría del $24 \%$. El paciente a los 30 días, había mejorado marcadamente su babeo en un $60 \%$, siendo este observado por el entorno del paciente, el cual refería su marcada satisfacción por el procedimiento realizado, su frecuencia era de 2 y la severidad de 1, su EVA de 28.

\section{Paciente 5}

Paciente de 13 años de edad, diagnosticado con Parálisis Cerebral tipo diparesia espástica asociado a un retardo mental leve con un buen grado de colaboración, las quejas por hipersalivación perturbaban moderadamente la auto confianza del paciente, así como su alimentación en forma moderada a severa. La sialorrea era frecuentemente con una frecuencia grado 3 y severidad grado 3, como resultado del babeo realizaba un cambio al día de polo (durante la alimentación) y ocasionalmente presentaba enrojecimiento de la piel. Escala análoga visual 60. Fueron aplicadas 5 unidades de toxina en glándula submaxilar y parotida de cada lado, obteniéndose una clara respuesta terapéutica a los 3 días de tratamiento con una mejoría del $34 \%$. El paciente a los 30 días, había mejorado marcadamente su babeo en un $74 \%$, siendo este observado por el entorno del paciente, el cual refería su marcada satisfacción por el procedimiento realizado, su frecuencia era de 2 y la severidad de 1, su EVA de 28.

\section{Paciente 6}

Paciente de 6 años de edad, diagnosticado con Parálisis Cerebral tipo cuadriparesia espástica. las quejas por hipersalivación perturbaban moderadamente el estado anímico del paciente, así como su desenvolvimiento en el colegio. La sialorrea era frecuentemente con una severidad grado 3 y frecuencia grado 3, clasificado como moderado, como resultado del babeo realizaba el cambio de 1 polo al día, presentaba enrojecimiento leve de la piel ocasionalmente, Escala análoga visual inicial fue 70. Fueron aplicadas 5 unidades de toxina en cada glándula parótida y submaxilar, obteniéndose una clara respuesta terapéutica a la primera visita de seguimiento con una mejoría del $29 \%$. El paciente a los 30 días, había mejorado marcadamente su babeo en un $64 \%$, siendo este observado por el entorno del paciente, el cual refería su marcada satisfacción por el procedimiento realizado, su frecuencia era de 2 y la severidad de 1, su EVA de 25.

\section{DISCUSIÓN}

La sialorrea, el derrame involuntario de la saliva y el flujo de saliva por la boca es extremadamente común en niños con Parálisis Cerebral (6). Mientras el babeo cesa entre los 15 a 18 meses de edad, puede ser visto en niños neurológicamente sanos hasta los 4 años (7). 
Rev Hered Rehab. 2019; 1:27-35.

Esta sialorrea producida por un control oral motor inadecuado en niños con Parálisis Cerebral. La secreción de saliva en individuos sanos es de 1 a 1.5 Lt/día, y de este un $45 \%$ se produce en las parótidas y $45 \%$ en submaxilares y $10 \%$ sublinguales y salivales menores, su importante contribución en la secreción de saliva (90\%) la hace un objetivo asequible e idóneo por su fácil acceso para tratar la sialorrea con toxina botulínica $\mathrm{A}(8-12)$.

Este síntoma puede ser potencialmente discapacitante y los niños neurológicamente afectados especialmente con Parálisis cerebral, están frecuentemente afligidos, aunque no es una "amenaza de vida", puede tener implicancias psicosociales tanto para el niño como para su familia, siendo estigmatizados por lo que actuaría además como una barrera física y emocional, para su relación en el colegio, así como en su vida cotidiana.

$\mathrm{Su}$ severidad y frecuencia pueden variar por diversos factores, como el grado de disfunción cognitiva y compromiso neurológico de cada paciente (13-16).

La sialorrea disminuyó significativamente en cada observación con un $95 \%$ de confianza y una $\mathrm{p}=0.000<0.05$ (Prueba de Friedman), es decir que la sialorrea disminuye cada día que pasa con la toxina botulínica.

Nuestros resultados muestran que la inyección de toxina botulínica A por vía transcutanea con apoyo de ultrasonido en un procedimiento beneficiosos y útil para tratar sialorrea.

Por nuestra parte no hemos observado ningún efecto adverso, lo que muestra que se trata de un procedimiento relativamente seguro cuando es realizado por Fisiatras con experiencia en el manejo de toxina botulínica A.

Se observó un mejor resultado clínico en aquellos pacientes con sialorrea menos severa que experimentaron una mejoría del $66 \%$. El inicio del efecto de la toxina varió con los pacientes siendo este de 3 a 7 días.

Una de las recomendaciones del presente trabajo seria que se debe incluir la aplicación de toxina botulínica $\mathrm{A}$ en el arsenal de tratamiento para la sialorrea en niños afectados neurológicamente.
Pensamos que la Escala Análoga Visual es un método objetivo y barato para evaluar la respuesta terapéutica al tratamiento, dada la variabilidad en la presentación clínica; mientras que la sialorrea fue descrita como severa para unos otros no notan al babeo como un aspecto mayor. Otros procedimientos empleados por otros autores han sido desde el calculo del número de toallas usados cada día, peso de rollos y hasta gamagrafía de glándulas salivales.

La aplicación de toxina botulínica A es efectivo y rápido para tratar la sialorrea en niños con sialorrea moderada severa de una manera potencialmente segura y promisoria, mejorando calidad de vida, así como elevando la autoestima del paciente, logrando una mejor aceptación del entorno y facilitando la atención de estos pacientes.

\section{Correspondencia:}

Emma Rivera La Plata

Correo electrónico: emmariveralp@yahoo.es

\section{REFERENCIAS BIBLIOGRAFICAS}

1. Bathia K, Münchau A, P Brown P. Botulinum toxin is a useful treatment in excesive drooling in saliva. $\mathrm{J}$ Neurol Neurocirugía Psiquiatría.1999;67 (5):697.

2. Bothwell J, Clarke K, Dooley J, et al.Botulinum toxin a as treatment for excessive drooling in children. Pediatr Neurol 2012;27(1):18-22.

3. Bushara K. Sialorrhea in amyotrofic lateral sclerosis: a hypothesis of a new treatment: botulinum toxin A injections of the parotid glands. Med Hypotheses. 1997; 48: 337-339.

4. Crysdale WS, McCann C, Roske L, Joseph M, Semenuk D, Chait P. Saliva control issues in the neurologically challenged. A 30 year experience in team management. Int $\mathrm{J}$ Pediatr Otorhinolaryngol. 2006; 70 (3): 519-527.

5. Dana L, Tilton A. Clinical study of botulinum-A toxin in the Treatment of sialorrhea in children with Cerebral Palsy. Laryngoscope 2012;112:73-81.

6. Carod Artal FJ. Tratamiento de la sialorrea en enfermedades neurológicas mediante inyecciones transcutáneas de toxina botulínica tipo A en las glándulas parótidas. Neurología. 2003; 18 (5): 280284

7. Giess R, Naumann $M$, Werner E, et al. Injections of botulinum toxin A into the salivary glands improve sialorrhea in amyotrophic lateral sclerosis. J. Neurol Neurosurg Psychiatry. 2009; 69:121-123.

8. López LJ, Castro A. Toxina botulínica: Aplicaciones terapéuticas. Barcelona: Masson; 2003

9. Porta M. Treatment of sialorrhoea with ultrasound 
Rev Hered Rehab. 2019; 1:27-35.

guided botulinum toxin type A injection in patients with neurological disorders. J Neurol Neurocirugía Psiquiatría. 2001; 70(4):538-540.

10. Lim M, Mace A, Reza N, Sandhu G. Botulinum toxin in the management of sialorrhoea: a systematic review. Clin Otolaryngol. 2006; 31: 267-272.

11. Bax M, Goldstein M, Rosenbaum P, et al. Executive Committee for the Definition of Cerebral palsy. Proposed defi nition and classification of cerebral palsy. Dev Med Child Neurol. 2005; 47 (8): 571-576.

12. Tahmassebi JF, Curzon ME. Prevalence of drooling in children with cerebral palsy attending special schools. Dev Med Child Neurol. 2003; 45 (9): 613617.
13. Brei TJ. Management of drooling. Semin in Pediatr Neurol. 2003; 10 (4): 265-270.

14. Brin MF. Basic and clinical aspects of botox. Toxicon. 2009; 54 (5): 676-682.

15. Jongerius PH, Van Den Hoogen FJ, Van Limbeek J, Gabreëls FJ, van Hulst K, Rotteveel JJ. Effect of botulinum toxin in the treatment of drooling: a controlled clinical trial. Pediatrics. 2004; 114 (3): 620-627.

16. Tan EK. Botulinum toxin treatment of sialorrhea: comparing different therapeutic preparations. Eur J Neurol. 2006; 13 (Supp11): 60-64.

Anexo1. Ficha de evaluación

NOMBRE
DIAGNOSTICO:
DOSIS:

CUIDADOR:

\section{ESCALA DE CLASIFICACION DE BABEO}

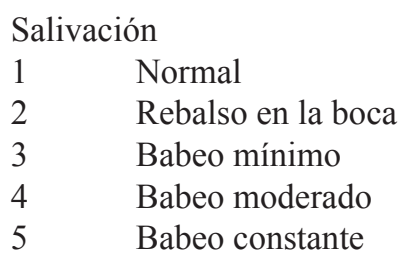

Cambio de polo o babero en un día como resultado del babeo excesivo

$1 \quad$ Ninguno

2 Un cambio por día de babero o polo

3 2-3 cambios por día de babero/polo

$4 \quad 4-5$ cambios por día de babero/polo

6 o más cambios por día de babero/polo

Babeo mientras come.

$\begin{array}{ll}1 & \text { Ninguno } \\ 2 & \text { Mínimo, no interfiere con la comida } \\ 3 & \text { Moderado, interfiere leve con la comida } \\ 4 & \text { Mod. - sev., interfiere obvio con la comida }\end{array}$

Severo, impide la alimentación

Episodios de tos severa o ahogo.

1 Nunca

2 Ha ocurrido sólo una o dos veces

3 Ocurre ocasionalmente

$4 \quad$ Ocurre con frecuencia

Ocurre siempre/casi todos los días

Respiración ruda o "gorgoteo" causado por saliva excesiva.

$1 \quad$ Nunca

2 Ocasionalmente (1 epis/sem o 1 epis/día) 
Rev Hered Rehab. 2019; 1:27-35.

3 Frecuente (mayor o igual a 2 episod/día)

4 Constante

Grado de irritación de la piel (cara/cuello) debido al babeo

1 Ninguno

2 Enrojecimiento leve, ocasionalmente

3 Enrojecimiento leve, siempre

4 Enrojecimiento moderado, siempre

Enrojecimiento severo, ocasionalmente excoriación.

Halitosis (mala respiración)

1 Ninguna

2 Halitosis leve

$3 \quad$ Halitosis moderada

$4 \quad$ Halitosis moderada-severa

$5 \quad$ Halitosis severa

Auto confianza del paciente secundaria al babeo

El babeo no afecta su confianza

El babeo causa leve perturbación

El babeo causa perturbación moderada

El babeo causa perturbación severa

Respuesta pública al paciente y el babeo

Normal, no se asusta

Mínima, levemente renuente a tener contacto físico con mi niño

Moderadamente renuente a tener contacto físico con mi niño

Moderado-severo, extremadamente renuente a tocar a mi niño

Severa, nunca tocan a mi niño.

Comodidad en el cuidado para el paciente en relación al babeo

No hay problema

Inconveniencia leve

Inconveniencia moderada

Inconveniencia severa

¿Cuán limitante es el babeo del paciente en las actividades fuera de la casa?

Ninguna

Muy leve

Leve

Moderada

Severa

Sobre todo: Cuán inconveniente es para usted (cuidante) por la excesiva saliva y/o babeo de su niño?

No le incomoda en absoluto

Le incomoda un poco

Le incomoda más que un poco, pero no mucho

Le incomoda mucho

Le incomoda extremadamente

Sobre todo: ¿Cuánto cree usted que se incomoda su niño como resultado del babeo excesivo?

No se incomoda en absoluto

Se incomoda más que un poco, pero no mucho

Se incomoda mucho

Se incomoda demasiado

ESCALA DE CLASIFICACIÓN DE LOS PARIENTES DE LA FRECUENCIA Y SEVERIDAD.

Frecuencia.

$1=$ Nunca 
Rev Hered Rehab. 2019; 1:27-35.

$2=$ Ocasionalmente (no todo el día)

$3=$ Frecuentemente (parte del día)

$4=$ Constantemente

Severidad

$1=$ Seco (ningún babeo)

$2=$ Mediano (sólo labios mojados)

$3=$ Moderado (labios y babero mojados)

$4=$ Severo (babeo que se extiende a la ropa)

$5=$ Profuso (manos, cubeta y objetos mojados)

SIALORREA: ESCALA VISUAL ANÁLOGA,

V.A.S.

$0-20$

$21-40$

$41-60$

61-80

$81-100$
Salivación

Normal

Rebalce de Boca

Babeo mínimo

Babeo moderado

Babeo constante.

\section{ESCALA DE CLASIFICACION POST-INYECCION}

\section{Escala de clasificación de nivel de satisfacción}

Fecha:

Sobre todo, ¿cómo tiene el babeo desde la inyección con Botox?

Marcadamente peor

Moderadamente peor

Levemente peor

No cambios

Leve mejoría

Mejoría moderada

Mejoría marcada

Si ha visto mejoría, cuánto ha mejorado para usted?

¿Cómo podría clasificar su nivel de satisfacción con el tratamiento para el babeo?

Marcadamente insatisfecho

Moderadamente insatisfecho

Levemente insatisfecho

Levemente satisfecho

Moderadamente satisfecho

Marcadamente satisfecho

¿Se colocaría usted nuevamente las inyecciones de Botox?

Sí

No 